\title{
Dual purpose passive screen for simultaneous display and imaging
}

Shoaib R. Soomro, Erdem Ulusoy, Muhsin Eralp, Hakan Urey

Shoaib R. Soomro, Erdem Ulusoy, Muhsin Eralp, Hakan Urey, "Dual purpose passive screen for simultaneous display and imaging," Proc. SPIE 10126, Advances in Display Technologies VII, 101260N (16 February 2017); doi: $10.1117 / 12.2251127$

SPIE. Event: SPIE OPTO, 2017, San Francisco, California, United States 


\title{
Dual Purpose Passive Screen for Simultaneous Display and Imaging
}

\author{
Shoaib R. Soomro*, Erdem Ulusoy, Muhsin Eralp, Hakan Urey \\ Koç University, Electrical Engineering Department, Optical Microsystems Laboratory, Istanbul, \\ Turkey
}

\begin{abstract}
Advanced imaging and display techniques are widely explored for realistic content capture and visualization but cannot fully follow the miniaturization and mobility trends in technology. Wide field-of-view displays require large surfaces and image capture requires separate installation of cameras having separate footprints and perspective views. Here we propose a novel, portable dual purpose passive screen that can simultaneously facilitate display and imaging with unprecedented features and performance. The optical design of the screen is presented. A prototype of the dual-purpose screen paired with a camera and a low power mobile projector is demonstrated. The developed screen has size of $28 \times 21 \mathrm{~cm}^{2}$ to facilitate capture of eye contacted perspective view and displays high-quality images with high-brightness $\left(>100 \mathrm{~cd} / \mathrm{m}^{2}\right)$ using only 15 lumen pico projector.
\end{abstract}

Keywords: Displays, Imaging, retro-reflective materials, Projection screens, Multipurpose screens

\section{INTRODUCTION}

Realistic content recording and visualization is crucial for human-computer interaction. Display technologies are quite advanced but mobile displays (e.g, smart phones) and emerging augmented reality headsets have small screens and offer limited field-of-view. Projection displays can offer wide field-of-view using only a small display engine but a projection surface is needed and the luminance is limited due to low lumen output of the projectors. The imaging technologies, on the other hand, are always considered as separate from display screen and cannot provide direct eye-contact for telepresence applications. A non-transparent Lambertian screen provides isotropic luminance in full hemi-sphere and is considered to have the luminance gain of 1. High optical gain is important for battery operated low-lumen output mobile devices and the gain can be improved by selectively scattering projected light using micro-lens arrays (MLA) ${ }^{1}$ or specially engineered scattering surfaces ${ }^{2}$. Very high gain screens are constructed using retro-reflective surfaces, which reflect projected light towards the source ${ }^{3}$. Most of the mobile devices used for telepresence (i.e. smartphone, laptops, tablets) have an imaging module to capture the user and its surroundings. Due to the distinct footprints of the display and imaging modules, such devices provide wrong camera perspective and cannot provide direct eye contact for telepresence applications ${ }^{4}$. The issue is partly addressed by performing post processing on captured images to correct the facial orientation, but such techniques require additional depth sensor for pose estimation ${ }^{5}$ and involve computationally expensive algorithms ${ }^{6}$.

In this paper, we propose a dual-purpose screen for imaging and display, which simultaneously acts as high gain projection screen and virtual perspective camera. The proposed screen is based on multiple optical surfaces arranged in the form of single integrated surface. The top surface of the screen is a micro-patterned retro-reflective film with adjustable optical gain and transparency, the bottom surface of the screen is a mirror, which creates a catadioptric imaging configuration and facilitates virtual image capture providing direct eye contact. The mirror surface on the screen forms a virtual perspective view of the scene, which is captured with a camera facing towards the screen. An additional polarizer surface is sandwiched between the top and the bottom surfaces, which facilitates the seamless separation of imaging and display functions.

\section{CONCEPT OF THE SYSTEM}

The system consists of dual purpose screen used together with a camera and pico projector fixed at the working distance from screen as illustrated in Figure 1. The camera records the perspective view by capturing the reflection of scene including viewer through the screen, while at the same time projector illuminates the screen with the projected content.

Advances in Display Technologies VII, edited by Liang-Chy Chien, Tae-Hoon Yoon, Sin-Doo Lee,

Proc. of SPIE Vol. 10126, 101260N - @ 2017 SPIE · CCC code: 0277-786X/17/\$18 · doi: 10.1117/12.2251127 
Due to the retro-reflective nature of screen, the viewer can see the bright content displayed on the screen, when standing in the close vicinity of projector. The proposed screen is composed of three different optical surfaces, where the combined functionality of all three surfaces performs display and imaging operations at the same time.

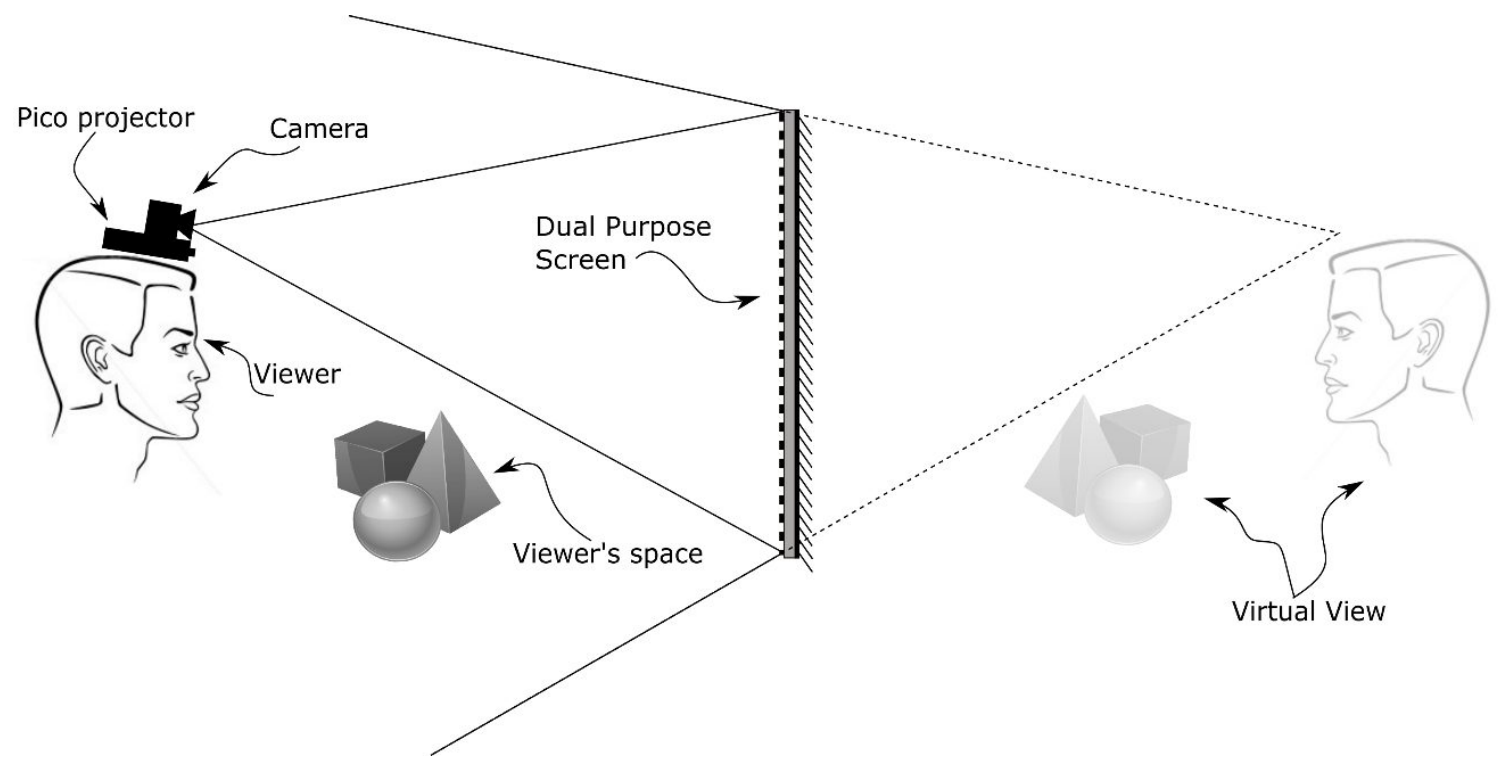

Figure 1:Conceptual illustration of the system, showing the image formation process and dual functionality of screen when used with a camera and pico projector.

The top surface of the screen is a display medium, which makes use of retro-reflective microspheres in the shape of micro-patterns to direct the light towards its source with narrow scattering. A $100 \%$ fill-factor retro-reflective surface can provide luminance gain of $>100$ within the viewing area ${ }^{3,7}$. The amount of retro-reflective material on an optically clear surface can be controlled to create see-through and retro-reflective regions. We design the top surface with $25 \%$ fillfactor to have enough luminance gain, which leaves us $75 \%$ area of screen to be used for imaging purpose. To combine the display surface with the imaging surface and minimize structural artifacts, the pitch size of micro-pattern is made small enough to be irresolvable by naked eye at a typical working distance from the screen. We used a pitch size of $200 \mathrm{um}$ to create micro-patterns on clear substrate. We use half-shell aluminum coated high refractive index microspheres to create retro-reflective patterns ${ }^{8}$. The microspheres based retro-reflective surface spreads incident light towards source in the shape of angular cone, which depends on the structural and material properties of microspheres. We use microspheres providing the cone angle of about $2^{\circ}{ }^{8,9}$. The perceived luminance of display increases gradually when viewer moves close to the projector.

The bottom surface of the screen is an imaging surface, which is based on the catadioptric configuration ${ }^{10}$. The surface consists of highly reflective flat mirror. The mirror surface creates the virtual image of the scene which is captured using a camera place close to projector, the camera capture the scene through the reflection of screen providing direct view from inside the screen as illustrated in Figure 1. A vertical polarizer surface is sandwiched between the retro-reflective surface and the imaging surface to create the dual-purpose screen. The polarizer absorbs the portion of the projector light blocking its reach from mirror. On the other hand, unpolarised ambient light from the scene is reflected off the screen as purely polarized in vertical direction. Camera having vertical polarizer in front, blocks the horizontally polarized light from the top surface and captures the vertically polarized light only returned from the imaging surface. Thereby, the presence of the sandwiched polarizer sheet allows both the imaging and the display functions to be performed simultaneously and without interference or crosstalk.

\section{SCREEN ASSEMBLY AND EXPERIMENTAL SETUP}

The screen assembly involved three stages. First, a transparent retro-reflective surface was fabricated, then a reflective surface was constructed, and finally, both surfaces were combined using a polarizer film in between. 


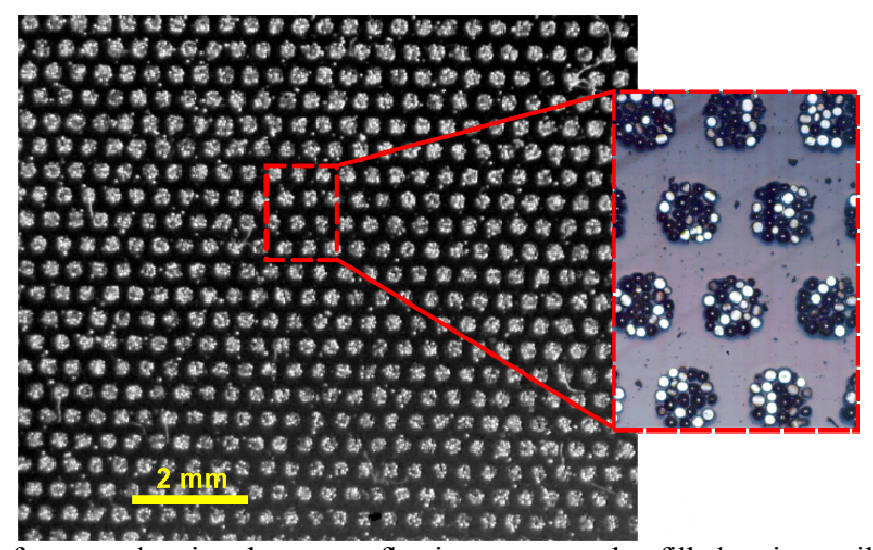

Figure 2: Shows the closed view of screen, showing the retro-reflective pattern and unfilled regions utilized for imaging.

The display surface was fabricated by patterning an optically clear substrate with retro-reflective microspheres ${ }^{8}$. A thin layer of optically clear adhesive was formed on the substrate to deposit the microspheres. A negative mask of pattern design was used to transfer the microspheres. The mask was made of stainless steel and had perforations to shape the retro-reflective microspheres on the substrate. The mask had only $25 \%$ perforated area, which resulted the $75 \%$ transparency on substrate. A large sized $\left(28 \times 21 \mathrm{~cm}^{2}\right)$ highly reflective mirror was used as imaging surface. The linear polarizer film was sandwiched between top display surface and bottom mirror surface and all three surfaces were bonded together using optical adhesives to create a portable screen. A close view of developed screen showing the retroreflective micro-patterns and mirror regions is shown in Figure 2. The microscopic view of screen shows that only a fraction of microspheres provides retro-reflectivity, due to their random orientation across screen. Figures 3 shows the experimental setup, where a camera and a pico projector placed at $60 \mathrm{~cm}$ distance and facing towards the screen are arranged. Imaging and display features of the screen were tested by using a $3.2 \mathrm{MP}$ camera ${ }^{11}$ and a 15 lumen (after a polarizer) laser sourced mobile projector ${ }^{12}$.

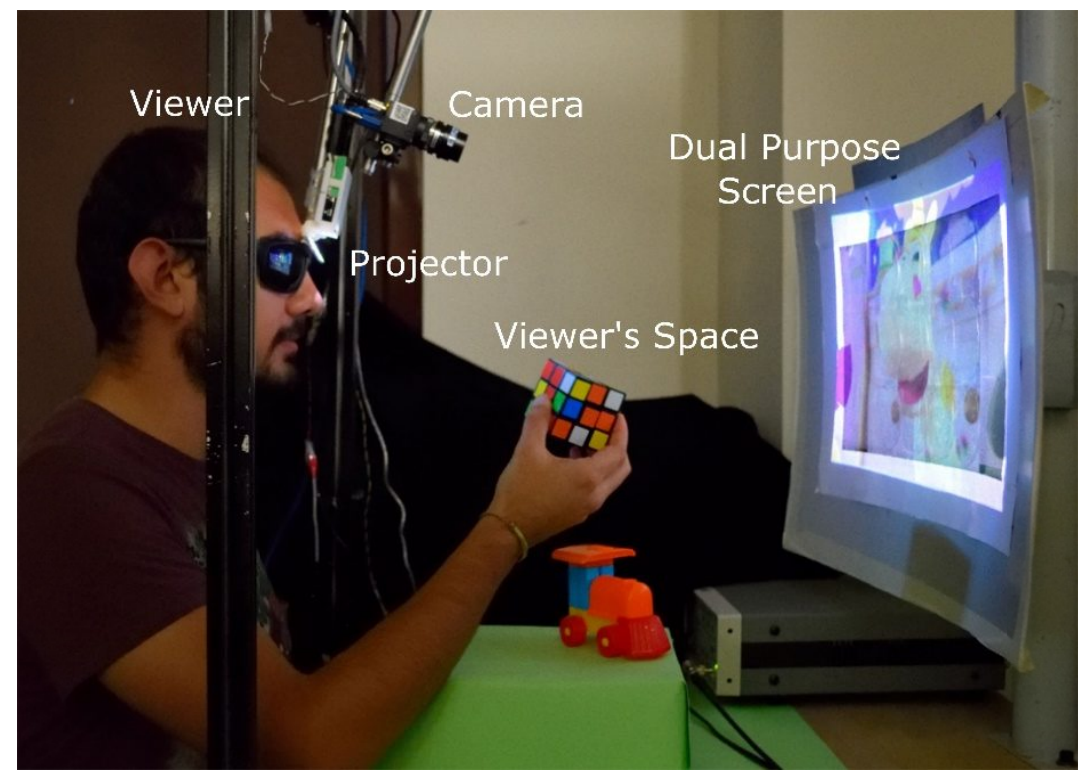

Figure 3: Shows the experimental setup consisting of dual purpose screen, a camera and projector. The screen displays the bright content, while at the same time, the reflection of scene is recorded using the camera.

\section{RESULTS AND DISCUSSION}

Display performance of the developed screen was evaluated by measuring the luminance of screen using a photometric camera $^{13}$ and a pico projector directly across the screen at the calibrated camera distance of $120 \mathrm{~cm}$ as shown in Figure 
4(a). The distance between the optical axis of the photometric camera and the projector was varied during the experiment to measure the luminance of screen for various eye positions. Further, the luminance gain of the screen was computed using following expression:

$$
\mathrm{G}=\frac{\mathrm{L}_{\mathrm{s}}}{\mathrm{L}_{\mathrm{d}}}
$$

When $L_{s}$ and $L_{d}$ are the luminance values for proposed screen and regular diffusing screen (i.e. Lambertian scatterer) respectively. The position of photometric camera (eye position) was changed between $0-5 \mathrm{~cm}$ with respect to projector and luminance values were recorded.
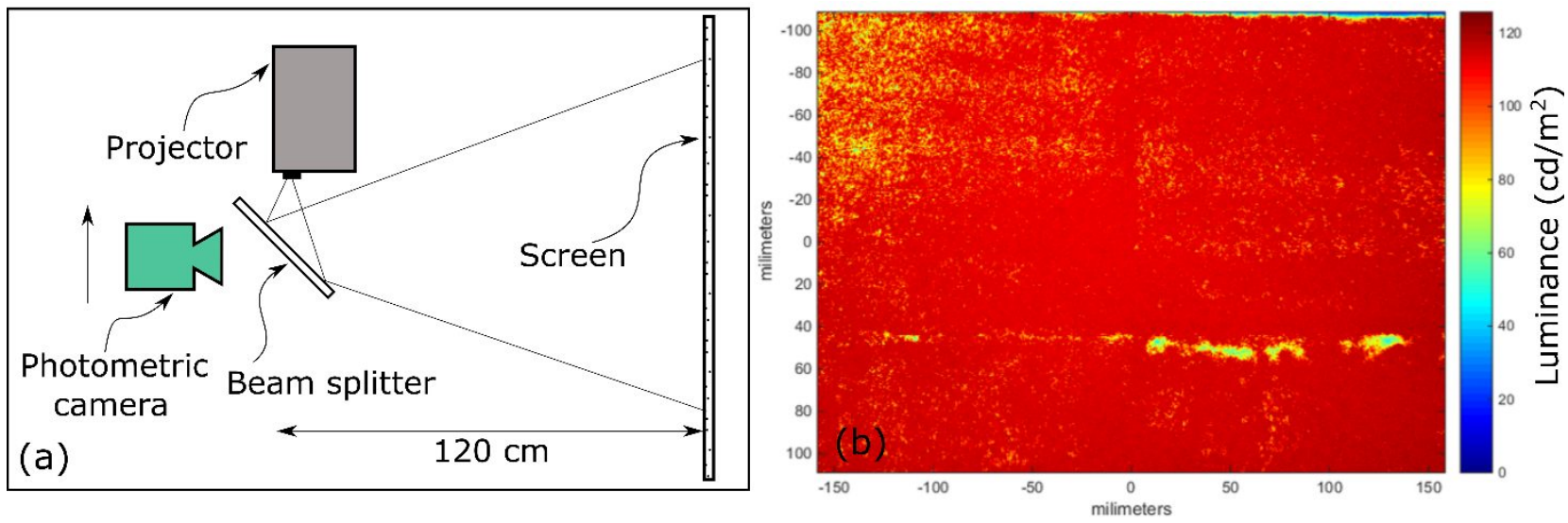

Figure 4:(a) Illustrates the arrangement projector and photometric camera to measure the luminance of screen, and (b) Shows the luminance of screen when distance between camera and projector is $0 \mathrm{~mm}$.

Table 1 shows luminance and optical gain of screen. The results showed significant luminance when the viewer is close to projector. The luminance and optical gain decline sharply with distance from the projector. Changing the microspheres size, shape, and refractive index can increase the angular spread of the light. The screen also show uniform luminance across the screen as seen in Figure 4(b)

Table 1: Screen luminance and gain for various eye positions

\begin{tabular}{|l|c|c|}
\hline \multicolumn{1}{|c|}{ Eye-to-Projector Distance } & $\begin{array}{c}\text { Luminance } \\
\left(\mathbf{c d} / \mathbf{m}^{\mathbf{2}}\right)\end{array}$ & Gain \\
\hline $00 \mathrm{~mm}$ (Through beam splitter) & 110 & 12 \\
\hline $10 \mathrm{~mm}$ & 380 & 41 \\
\hline $20 \mathrm{~mm}$ & 136 & 15 \\
\hline $30 \mathrm{~mm}$ & 60 & 6.5 \\
\hline $35 \mathrm{~mm}$ & 29 & 3 \\
\hline $40 \mathrm{~mm}$ & 19.6 & 2 \\
\hline $50 \mathrm{~mm}$ & 14.4 & 1.5 \\
\hline
\end{tabular}

The content displayed on screen is viewed through linearly polarized glasses passing the horizontally polarized light only. Figure 5(a) shows the content displayed on the screen as seen by a viewer standing close to the projector. The polarizer completely absorbs the reflected light and provides the high display contrast with black background. 

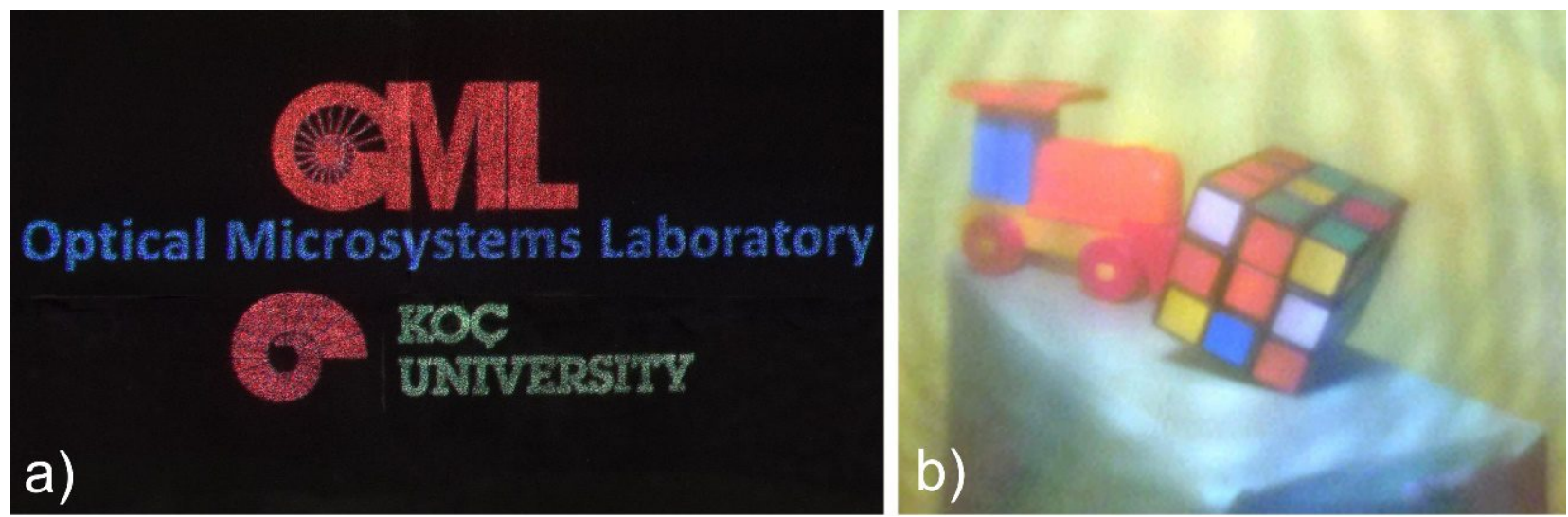

Figure 5: (a) Content displayed on screen using pico-projector and seen by viewer though polarized glassed and, (b) image of the viewer's space captured using screen reflection.

The imaging capability of the screen is limited by the pixel size and resolution of the capture camera and the best imaging performance is obtained when the camera is focused at the mirror image and the retro-reflective pattern is highly blurred. Imaging using the proposed screen was demonstrated by capturing the raw image of the scene through the screen reflection, while the displayed content was blocked by the camera. Figure 5(b) shows the image of the object placed in front of the screen (viewer's space) as captured through screen. The image quality of the screen can be further improved by using higher resolution cameras by optimizing the pattern pitch, fill-factor and efficiency of the retroreflective surface

\section{CONCLUSION}

We proposed and demonstrated a dual-purpose screen for concurrent imaging and display. The developed screen provides high luminance $\left(>100 \mathrm{~cd} / \mathrm{m}^{2}\right)$ when used with a low power 15 lumens mobile projector. Mirror surface was utilized for creating the imaging surface. The developed imaging surface provides direct view of the real-world scene from the perspective of screen, when paired with a camera facing towards screen. We combined both imaging and display surfaces in the shape of single integrated screen using polarization selective layer. Various screen geometries and configurations can be further explored. A detailed comparison of capture quality with standard cameras can also be performed. The proposed screen can be used for telepresence applications to provide direct eye-contact.

\section{ACKNOWLEDGMENTS}

This research is sponsored by European Research Council Advanced Grant (ERC-AdG) Wear3D Project No: 340200. We thank Sven Holmstrom for helpful discussions.

\section{REFERENCES}

[1] Hedili, M.K., Freeman, M.O., Urey, H., "Microlens array-based high-gain screen design for direct projection headup displays." Applied optics 2013; 52:1351-1357.

[2] Zuchowski, L., "Controlled gain projection screen." United States patent US 6,483,643 2002.

[3] Hua H., Gao, C., Rolland, J.P., "Imaging properties of retro-reflective materials used in head-mounted projective displays (HMPDs)", Proc. SPIE 4711, Helmet- and Head-Mounted Displays VII, 194(2002).

[4] Soomro, S.R., Ulusoy, E. and Urey, H., "Decoupling of Real and Digital Content in Projection-based Augmented Reality Systems Using Time Multiplexed Image Capture", Journal of Imaging Science and Technology 60 (1), (2017). 
[5] Kuster, C., Popa, T., Bazin, J. C., Gotsman, C., Gross, M. "Gaze correction for home video conferencing", ACM Transactions on Graphics (TOG), 31 (6), 174 (2012).

[6] Giger, D., Bazin, J. C., Kuster, C., Popa, T., \& Gross, M., "Gaze correction with a single webcam", IEEE International Conference on Multimedia and Expo (ICME), 1-6 (2014).

[7] Lloyd J., "A brief history of retroreflective sign face sheet materials". The Retroreflective Equipment Manufacturers Association: Lancashire, UK http://www.rema.org.uk/ (accessed January 2016).

[8] Soomro, S.R. and Urey, H., "Design, fabrication and characterization of transparent retro-reflective screen." Opt. Exp. 24(21), 24232-24241 (2016).

[9] Soomro, S.R. and Urey H., "Retro-reflective Characteristics of Transparent Screen for Head Mounted Projection Displays." OSA Frontiers in Optics, FTu5A-2 (2016).

[10] Gluckman J., Nayar S.K., "Rectified catadioptric stereo sensors", IEEE Transactions on Pattern Analysis and Machine Intelligence 24, 224-236 (2012).

[11] Pointgrey Flea3 Camera. https://www.ptgrey.com/flea3-32-mp-color-usb3-vision-sony-imx036-camera (accessed December 2016)

[12] Sony MP-CL1 mobile projector, http://www.sony.co.in/electronics/projector/mp-cll (accessed July 2016).

[13] Radiant Vision Systems, PM-1001 Prometric Colorimeter Camera, calibrated for $120 \mathrm{~cm}$. Available from: http://www.radiantvisionsystems.com. 